\title{
CONTROLLING THE GENERIC FORMAL FIBER OF LOCAL DOMAINS AND THEIR POLYNOMIAL RINGS
}

\author{
PEIHONG JIANG, ANNA KIRKPATRICK, S. LOEPP, \\ SANDER MACK-CRANE AND S. TRIPP
}

\begin{abstract}
Let $T$ be a complete local ring with maximal ideal $M, C$ a countable set of incomparable prime ideals of $T$, and $B_{1}$ and $B_{2}$ sets of prime ideals of $T\left[\left[x_{1}, \ldots, x_{n}\right]\right]$ with cardinality less than that of $T$. We present necessary and sufficient conditions for the existence of a local domain $A$ with completion $T$, such that the generic formal fiber of $A$ has maximal elements equal to the ideals in $C$ and the generic formal fiber of $A\left[x_{1}, \ldots, x_{n}\right]_{\left(M \cap A, x_{1}, \ldots, x_{n}\right)}$ contains every element of $B_{1}$ but no element of $B_{2}$. If $T$ has characteristic 0 , we present necessary and sufficient conditions for the existence of an excellent local domain $A$ with the above properties.
\end{abstract}

1. Introduction. Let $A$ be a (Noetherian) local ring with maximal ideal $\mathbf{m}$ and $\mathbf{m}$-adic completion $\widehat{A}=T$. If $Q$ is a prime ideal of $A$, then the formal fiber of $A$ at $Q$ is defined to be $\operatorname{Spec}\left(\widehat{A} \otimes_{A} k(Q)\right)$ where $k(Q)$ is the field $A_{Q} / Q A_{Q}$. As the formal fibers of a ring are known to encode important geometric information about the ring (see, for example, [9]), we want to understand the general behavior of formal fibers. However, many open questions remain. For example, if $T$ is a complete (Noetherian) local ring and $G$ a set of prime ideals of $T$, it is unknown when there exists a (Noetherian) local integral domain $A$ such that the completion of $A$ is $T$ and such that the formal fiber of $A$ at the prime ideal (0) is exactly the set $\left\{\left(P \otimes_{A} K\right) \mid P \in G\right\}$ where $K=k((0))$ is the quotient field of $A$. We note that the answer is, in fact, known in the case that $G$ has only countably many maximal elements with respect to inclusion, and we will discuss that result in more detail later (see Theorem 1.1).

The authors thank the National Science Foundation for support of this research via grant No. DMS-0850577. The last author thanks the Finnerty Fund at Williams College for the financial support of his research.

Received by the editors on September 13, 2013, and in revised form on March 31, 2014. 
Consider the following more general open question. Let $A \rightarrow S$ be a flat extension of (Noetherian) local rings. What is the relationship between the formal fibers of $A$ and the formal fibers of $S$ ? If we knew the answer to this question, we could then gain insight into the geometric behavior of $S$ (or $A$ ) assuming we understand something about the geometric behavior of $A$ (or $S$ ). Sadly, however, very little is known about the behavior of formal fibers for flat extensions. Suppose, for example, that $P$ is a nonmaximal prime ideal of $A$, and let $S$ be the localization of $A$ at $P$. Almost nothing is known about the relationship between the formal fibers of $A$ and the formal fibers of $S$ in this case. In fact, even if $A$ is a complete integral domain, we do not know the dimension of the ring $\widehat{S} \otimes_{S} K$ where $K$ is the quotient field of $S$. Heinzer, Rotthaus and Sally have informally conjectured that, in this case, if the height of $P$ is at least two, then the dimension of the ring $\widehat{S} \otimes_{S} K$ is two less than the height of $P$.

More is known in the case where $S$ is a polynomial ring over $A$. Specifically, let $\mathbf{m}$ be the maximal ideal of $A$, and let $S=$ $A\left[x_{1}, \ldots, x_{n}\right]_{\left(\mathbf{m}, x_{1}, \ldots, x_{n}\right)}$, where $x_{1}, x_{2}, \ldots, x_{n}$ are indeterminates. In [6], Loepp and Rotthaus show that if $n=1$ and $A$ is a universally catenary (Noetherian) local integral domain such that the dimension of the ring $\widehat{S} \otimes_{S} k((0))$ is equal to the dimension of $A$, then the dimension of the ring $\widehat{A} \otimes_{A} k((0))$ is exactly one less than the dimension of $A$. They also show that the converse of the above statement is not true. Along the same lines, in [7], Loepp and Weinberg show that, in the case where $A$ is an integral domain, it is possible to simultaneously control the formal fiber of $A$ at (0) and the formal fiber of $S=A\left[x_{1}, \ldots, x_{n}\right]_{\left(\mathbf{m}, x_{1}, \ldots, x_{n}\right)}$ at (0) (see Theorem 1.2). As an example, they construct an $A$ where the dimension of the ring $\widehat{A} \otimes_{A} k((0))$ is "small," but the dimension of the $\operatorname{ring} \widehat{S} \otimes_{S} k((0))$ is "large."

We note here that the results mentioned in the previous two paragraphs give insight into formal fibers at the zero ideal. It seems that even less is known about formal fibers at nonzero prime ideals.

In this paper, we consider the previously mentioned case where $A$ is an integral domain and $S=A\left[x_{1}, \ldots, x_{n}\right]_{\left(\mathbf{m}, x_{1}, \ldots, x_{n}\right)}$, and we improve the results of Loepp and Weinberg in [7]. For ease of notation, we will use $\underline{x}$ to represent the indeterminates $x_{1}, \ldots, x_{n}$. The generic formal 
fiber of $A$ is defined to be the formal fiber of $A$ at the zero ideal. In other words, if $\widehat{A}=T$ and the quotient field of $A$ is $K$, then the generic formal fiber of $A$ is $\operatorname{Spec}\left(T \otimes_{A} K\right)$. We can identify this spectrum with the set of prime ideals $P \in \operatorname{Spec} T$ such that $P \cap A=(0)$, and we will treat this as an alternate definition; that is, the generic formal fiber of a (Noetherian) local integral domain $A$ can be thought of as the set of prime ideals $P \in \operatorname{Spec} T$ such that $P \cap A=(0)$. Observe that the generic formal fiber of $A$ is determined by its maximal elements with respect to inclusion, for if $P^{\prime} \subseteq P \in \operatorname{Spec} T$ with $P \cap A=(0)$ then $P^{\prime} \cap A=(0)$ as well.

We are interested in proving the existence of (Noetherian) local domains $A$ with a specified completion and generic formal fiber, such that we can simultaneously restrict the generic formal fiber of $A[\underline{x}]_{(M \cap A, \underline{x})}$ (where $M$ is the maximal ideal of $\widehat{A}$ ). In Section 3, we present necessary and sufficient conditions for the existence of such a ring $A$ with various restrictions on the generic formal fiber of $A[\underline{x}]_{(M \cap A, \underline{x})}$. In each theorem, it is fairly straightforward to show that the conditions are necessary. To show that they are sufficient is far more difficult, and we accomplish this by constructing the desired ring. This construction is carried out in Section 2.

In this paper, all rings are commutative with unity. A ring with a single maximal ideal will be called quasi-local; we reserve the word local for a Noetherian ring with a single maximal ideal. When we say $(R, M)$ is a local ring, we mean $R$ is a local ring with maximal ideal $M$. We use $c$ to denote the cardinality of the continuum.

In [2], Charters and Loepp characterized complete local rings that are the completion of a local domain with generic formal fiber having finitely many maximal elements. In [1], Aiello, Loepp and Vu relaxed the condition of finitely many maximal elements to countably many maximal elements. We will make use of [1, Lemma 2.7] to allow countably many maximal elements in our theorems as well. We shall make extensive use of the techniques from $[2, \mathbf{1}]$ in our construction, and we repeat the main theorem from $[\mathbf{1}]$ here.

Theorem 1.1 ([1], Theorem 2.13). Let $(T, M)$ be a complete local ring with prime subring $\Pi$, and let $G \subset \operatorname{Spec} T$ be such that $G$ is nonempty and the number of maximal elements of $G$ is countable. Then there 
exists a local domain $A$ such that $\widehat{A}=T$ and the generic formal fiber of $A$ is exactly the elements of $G$ if and only if $T$ is a field and $G=\{(0)\}$ or the following conditions hold:

(i) $M \notin G$, and $G$ contains all the associated prime ideals of $T$.

(ii) If $Q \in G$ and $P \in \operatorname{Spec} T$ with $P \subseteq Q$, then $P \in G$.

(iii) If $Q \in G$ then $Q \cap \Pi=(0)$.

This theorem allows us to prove the existence of a local domain $A$ with a generic formal fiber of our choosing, as long as the generic formal fiber we specify has countably many maximal elements.

As mentioned above, the problem we attack in this paper is the existence of local domains $A$ such that we can specify the completion and generic formal fiber of $A$ and simultaneously restrict the generic formal fiber of $A[\underline{x}]_{(M \cap A, \underline{x})}$ (where $M$ is the maximal ideal of $\widehat{A}$ ). There are some obvious restrictions on the generic formal fiber of $A[\underline{x}]_{(M \cap A, \underline{x})}$. For $Q \in \operatorname{Spec} \widehat{A}[[\underline{x}]]$, if $Q \cap \widehat{A}$ is not in the generic formal fiber of $A$ then $Q$ can never be in the generic formal fiber of $A[\underline{x}]_{(M \cap A, \underline{x})}$. Similarly, if $Q \cap \Pi[\underline{x}]$ is not (0) (where $\Pi$ is the prime subring of $\widehat{A}$ ) then $Q$ can never be in the generic formal fiber of $A[\underline{x}]_{(M \cap A, \underline{x})}$. We are interested in controlling the prime ideals of $\widehat{A}[[\underline{x}]]$ whose fate is unknown, i.e., those whose intersection with $\Pi[\underline{x}]$ is $(0)$, and whose intersection with $\widehat{A}$ lies in the generic formal fiber of $A$.

In [7], Loepp and Weinberg give a partial solution to this problem, a version of which we repeat below.

Theorem 1.2 ([7], Theorem 13). Let $(T, M)$ be a complete local ring with prime subring $\Pi$. Suppose $|T / M| \geq c$ and that no integer of $T$ is a zero divisor. Let $P \in \operatorname{Spec} T$ and $B_{1} \subset \operatorname{Spec} T[[\underline{x}]]$ with $\left|B_{1}\right|<|T / M|$. Then there exists a local domain $A$ such that $\widehat{A}=T$, the generic formal fiber of $A$ is local with maximal ideal $P$, and the generic formal fiber of $A[\underline{x}]_{(M \cap A, \underline{x})}$ contains every element of $B_{1}$ if the following conditions hold.

(i) $P \neq M$ and $P$ contains all associated prime ideals of $T$.

(ii) $P \cap \Pi=(0)$.

(iii) If $Q \in B_{1}$ then $Q \cap T \subseteq P$.

(iv) If $Q \in B_{1}$ then $Q \cap \Pi[\underline{x}]=(0)$. 
Note that, although it is not explicitly stated in their paper, it is not difficult to show that these four conditions are in fact necessary as well.

Our Corollary 3.6 improves the above theorem by removing the hypothesis that $|T / M| \geq c$, relaxing the condition $\left|B_{1}\right|<|T / M|$ to $\left|B_{1}\right|<|T|$, and allowing the generic formal fiber of $A$ to have countably many maximal elements instead of just one.

In addition, given another set $B_{2} \subset \operatorname{Spec} T[[\underline{x}]]$ with $\left|B_{2}\right|<|T|$, our Theorem 3.1 gives necessary and sufficient conditions to achieve the above result and simultaneously ensure that no element of $B_{2}$ is in the generic formal fiber of $A[\underline{x}]_{(M \cap A, x)}$. This gives us still more control over the generic formal fiber of $A[\underline{x}]_{(M \cap A, \underline{x})}$.

A closely related problem, which we also address, is to construct excellent local domains with a specified generic formal fiber. Charters and Loepp in [2] accomplished this for complete local rings of characteristic 0 and generic formal fibers with finitely many maximal elements. In [1] Aiello, Loepp and $\mathrm{Vu}$ were able to relax the condition of finitely many maximal elements to countably many maximal elements. We state the relevant theorem from [1] here.

Theorem 1.3 ([1], Theorem 2.15). Let $(T, M)$ be a complete local ring containing the integers, and call its prime subring $\Pi$. Let $G \subset \operatorname{Spec} T$ be such that $G$ is nonempty and the set of maximal elements of $G$ is countable. Then there exists an excellent local domain $A$ with $\widehat{A}=T$ and such that $A$ has generic formal fiber exactly $G$ if and only if $T$ is a field and $G=\{(0)\}$ or the following conditions hold:

(i) $M \notin G$, and $G$ contains all the associated prime ideals of $T$.

(ii) If $Q \in G$ and $P \in \operatorname{Spec} T$ with $P \subseteq Q$, then $P \in G$.

(iii) If $Q \in G$, then $Q \cap \Pi=(0)$.

(iv) $T$ is equidimensional.

(v) $T_{P}$ is a regular local ring for all maximal elements $P \in G$.

For the existence of excellent local domains $A$ with restrictions on the generic formal fiber of $A[\underline{x}]_{(M \cap A, \underline{x})}$, Weinberg and Loepp in [7] give the following result. 
Theorem 1.4 ([7], Theorem 14). Let $(T, M)$ be a complete regular local ring with prime subring $\Pi$. Suppose $T$ has dimension at least two, contains the rationals, and has $|T / M| \geq c$. Let $P \in \operatorname{Spec} T$ and $B_{1} \subset \operatorname{Spec} T[[\underline{x}]]$ such that $\left|B_{1}\right|<|T / M|$. Then there exists an excellent regular local ring $A$ such that $\widehat{A}=T$, the generic formal fiber of $A$ is local with $P$ its maximal ideal and every element of $B_{1}$ is in the generic formal fiber of $A[\underline{x}]_{(M \cap A, \underline{x})}$ if the following conditions hold.

(i) $P \neq M$.

(ii) If $Q \in B_{1}$ then $Q \cap T \subseteq P$.

(iii) If $Q \in B_{1}$ then $Q \cap \Pi[\underline{x}]=(0)$.

Again, though it is not explicitly stated, it is not difficult to show that these conditions are necessary as well.

In Section 4, we use techniques from [2] to accomplish a similar result under much weaker hypotheses. Corollary 4.3 has the same conclusions as Theorem 1.4 except that the ring $A$ may not be regular local, and it improves the above result by removing the hypothesis that $T$ is a regular ring, removing the hypothesis that $|T / M| \geq c$, relaxing $\left|B_{1}\right|<|T / M|$ to $\left|B_{1}\right|<|T|$, and allowing the generic formal fiber of $A$ to have countably many maximal elements instead of one.

In addition, in Theorem 4.1 we give similar necessary and sufficient conditions to accomplish the above result and simultaneously specify a set $B_{2} \subset \operatorname{Spec} T[[\underline{x}]]$ with $\left|B_{2}\right|<|T|$ such that no element of $B_{2}$ is in the generic formal fiber of $A[\underline{x}]_{(M \cap A, \underline{x})}$.

2. Construction. In this section, we will construct an integral domain $A$ with the desired properties. Our construction builds upon the construction seen in [2] and includes modifications from [1]. The general idea of this construction is as follows. Starting with the prime subring of $T$, we adjoin a set of elements related to the ideals we want to keep outside the generic formal fiber of $A[\underline{x}]_{(M \cap A, \underline{x})}$ and then build up a chain of subrings. Each subring is created by adjoining elements of $T$ to the previous subring in a way that will eventually yield a subring whose completion is $T$. Each subring along the way also purposefully dodges all nonzero elements of the prime ideals in $G$ and $B_{1}$ so that they will be in the generic formal fiber of $A$ and $A[\underline{x}]_{(M \cap A, \underline{x})}$, respectively. 
The union of this chain of subrings is our local domain with desired completion and generic formal fiber properties.

Certain variable names will be used consistently throughout this paper, and we list them here to help the reader follow our construction. We will use $(T, M)$ to denote a complete local ring with prime subring $\Pi$. We will construct a local domain $A$ whose completion is $T$. The set $G$ contains prime ideals of $T$ that we would like to be exactly the generic formal fiber of $A$. The set $C$ contains prime ideals of $T$ that we would like to be the maximal elements of the generic formal fiber of $A$, that is, $C$ contains exactly the maximal elements of $G$. The sets $B_{1}$ and $B_{2}$ contain prime ideals of $T[[\underline{x}]]$; we want the ideals in $B_{1}$ to be in the generic formal fiber of $A[\underline{x}]_{(M \cap A, \underline{x})}$, and we want the ideals in $B_{2}$ not to be in the generic formal fiber of $A[\underline{x}]_{(M \cap A, \underline{x})}$.

The following proposition, from [5], will be used to show that the domain we construct has the completion we desire.

Proposition 2.1 ([5], Proposition 1). If $(A, M \cap A)$ is a quasi-local subring of a complete local ring $(T, M)$, the map $A \rightarrow T / M^{2}$ is onto, and $I T \cap A=I$ for every finitely generated ideal $I$ of $A$, then $A$ is Noetherian, and the natural homomorphism $\widehat{A} \rightarrow T$ is an isomorphism.

We will use Lemma 2.2 in various places throughout the construction, and state it here without proof.

Lemma 2.2. Let $T$ be an integral domain and $I$ a nonzero ideal of $T$. Then $|I|=|T|$.

We will use the following definition to simplify our work with cardinalities.

Definition. Let $S$ be a set. Then $\Gamma(S)=\sup \left(|S|, \aleph_{0}\right)$.

The following lemma, adapted from [3, Lemma 2.5], will be used to control the cardinality of unions of subrings and sets. 
Lemma 2.3 ([3], Lemma 2.5). Suppose $H$ is a set of sets and $\lambda$ a cardinal number. If $\Gamma(h) \leq \lambda$ for all $h \in H$, and $\Gamma(H) \leq \lambda$, then $\Gamma\left(\bigcup_{h \in H} h\right) \leq \lambda$.

The next lemma gives us a handle on the cardinality of the quotient of $T$ by any nonmaximal prime ideal.

Lemma 2.4 ([2], Lemma 2.3). Let (T, $M$ ) be a complete local ring of dimension at least one. Let $P$ be a nonmaximal prime ideal of $T$. Then $|T|=|T / P| \geq c$.

The following lemma is a more powerful version of the prime avoidance lemma, which will allow us to choose elements which are transcendental over subrings by avoiding cosets of algebraic elements. We use transcendental elements to ensure that our subrings avoid the ideals we want to put in the generic formal fiber.

Lemma 2.5 ([1], Lemma 2.7). Let $(T, M)$ be a complete local ring of dimension at least one. Let $C$ be a countable set of nonmaximal prime ideals of $T$, and let $D$ be a subset of $T$ such that $|D|<|T|$. Let $I$ be an ideal of $T$ such that $I \nsubseteq P$ for all $P \in C$. Then $I \nsubseteq \bigcup\{r+P \mid r \in D, P \in C\}$.

There are certain properties that all the subrings we construct must share, and for easy access we record them in the definition below.

Definition. Let $(T, M)$ be a complete local ring and $C$ a set of prime ideals of $T$. Let $B_{1}$ be a set of prime ideals of $T[[\underline{x}]]$. Suppose that $(R, R \cap M)$ is a quasi-local subring of $T$ such that $|R|<|T|, R \cap P=(0)$ for all $P \in C$, and $R[\underline{x}] \cap Q=(0)$ for all $Q \in B_{1}$. Then we call $R$ a small, $C$ and $B_{1}$ avoiding subring of $T$ and will denote it by SCBA-subring.

The following lemma, derived from [2, Lemma 2.5], enables us to adjoin a specific element of a quotient of $T$ while we are constructing our local domain $A$. We use this to obtain the property that $A \rightarrow T / J$ is onto for all ideals $J$ such that $J \nsubseteq P$ for all $P \in C$, which satisfies one hypothesis of Proposition 2.1 and therefore is a step toward 
showing that our constructed domain has the desired completion. It is also crucial to our proof that $A$ can be made excellent under certain circumstances.

Lemma 2.6. Let $(T, M)$ be a complete local ring of dimension at least one, $C$ a countable set of nonmaximal prime ideals of $T$, and $B_{1}$ a set of prime ideals of $T[[\underline{x}]]$ such that $\left|B_{1}\right|<|T|$ and for each $Q \in B_{1}$, $Q \cap T \subseteq P$ for some $P \in C$. Let $J$ be an ideal of $T$ such that $J \nsubseteq P$ for all $P \in C$. Let $R$ be an SCBA-subring of $T$ and $u+J \in T / J$. Then there exists an infinite SCBA-subring $S$ of $T$ such that $R \subseteq S \subseteq T$, $\Gamma(R)=\Gamma(S)$, and $u+J$ is in the image of the map $S \rightarrow T / J$. Moreover, if $u \in J$, then $S \cap J \neq(0)$.

Proof. Note that if $P \in C, R \cap P=(0)$, and so $R$ embeds in $T / P$, and a coset of $P$ being algebraic over $R$ is well defined. For each $P \in C$, let $D_{(P)}$ be a full set of coset representatives of the cosets $t+P$ that make $(u+t)+P$ algebraic over $R$. Similarly, $R[\underline{x}] \cap Q=(0)$ for each $Q \in B_{1}$, and so $R[\underline{x}]$ embeds in $T[[\underline{x}]] / Q$, and a coset of $Q$ being algebraic over $R[\underline{x}]$ is well defined. For each $Q \in B_{1}$, define $D_{(Q)}$ to be a full set of coset representatives of the elements $t+(Q \cap T)$ in $T /(Q \cap T)$ that make $(u+t)+Q$ in $T[[\underline{x}]] / Q$ algebraic over $R[\underline{x}]$. Note also for $t, t^{\prime} \in T, t+(Q \cap T)=t^{\prime}+(Q \cap T)$ if and only if $(u+t)+Q=\left(u+t^{\prime}\right)+Q$. The only if direction ensures that $D_{(Q)}$ is well defined, and the if direction ensures that the cardinality of $D_{(Q)}$ is the number of elements of $T[[\underline{x}]] / Q$ algebraic over $R[\underline{x}]$ (in particular, $D_{(Q)}$ is no bigger than this cardinality).

We know $|R|<|T|$ and $|T| \geq c$, so $|R[\underline{x}]|<|T|$. For the same reason (and the fact that every polynomial has finitely many solutions), $|R[\underline{x}]|=\left|D_{(P)}\right|<|T|$ for each $P \in C$ and $|R[\underline{x}]|=\left|D_{(Q)}\right|<|T|$ for each $Q \in B_{1}$. Similarly, note that the set of elements algebraic over $R$ has the same cardinality as $R$.

Let $D=\left(\bigcup_{P \in C} D_{(P)}\right) \cup\left(\bigcup_{Q \in B_{1}} D_{(Q)}\right)$. Now $\bigcup_{P \in C} D_{(P)}$ is a countable union of sets each with cardinality at most that of $R[\underline{x}]$, so by Lemma 2.3 , it has cardinality at most that of $R[\underline{x}]$. Also, $\bigcup_{Q \in B_{1}} D_{(Q)}$ is a union of $\left|B_{1}\right|$ sets, each of cardinality at most that of $R[\underline{x}]$. Letting $\lambda=\max \left(\left|B_{1}\right|,|R[\underline{x}]|\right)$, we use Lemma 2.3 to see that $\left|\bigcup_{Q \in B_{1}} D_{(Q)}\right| \leq \lambda<|T|$. Finally, we use Lemma 2.3 one last time to note $|D| \leq \lambda<|T|$. 
By construction, $D \subset T$. Now we can use Lemma 2.5 with $I=J$ and our $C$ and $D$. This gives us an element $y \in J$ such that $y \notin \bigcup\{r+P \mid r \in D, P \in C\}$. We claim that $S=R[u+y]_{(R[u+y] \cap M)}$ is the desired SCBA-subring. It is easy to see that $\Gamma(R)=\Gamma(S)$, and thus $|S|<|T|$.

Suppose that $f \in R[u+y] \cap P$ for some $P \in C$. Then $f=$ $r_{n}(u+y)^{n}+\cdots+r_{1}(u+y)+r_{0} \in P$ where $r_{i} \in R$. But, by avoiding the sets $D_{(P)}$, we chose $y$ such that $(u+y)+P$ is transcendental over $R$ in $T / P$. Therefore, $r_{i}+P=0+P$ (i.e., $r_{i} \in P$ ) for each $i$, but $r_{i} \in R$, also. Thus, $r_{i} \in R \cap P=(0)$, and $f=0$. Therefore, $R[u+y] \cap P=(0)$, and this implies $S \cap P=(0)$. Furthermore, if $u \in J$, then $u+y \in J$. Since $(u+y)+P$ is transcendental over $R$, we have that $S$ is infinite and $u+y \neq 0$. But $u+y \in S \cap J$, so $S \cap J \neq(0)$.

Now suppose that $g \in R[u+y][\underline{x}] \cap Q$ for some $Q \in B_{1}$. Note $R[u+y][\underline{x}]=R[\underline{x}][u+y]$ so we can write $g=s_{n}(u+y)^{n}+\cdots+s_{1}(u+$ $y)+s_{0} \in Q$ where $s_{i} \in R[\underline{x]}$. We chose $y \notin \bigcup\{r+P \mid r \in D, P \in C\}$, and so $(u+y)+Q \in T[[\underline{x}]] / Q$ is transcendental over $R[\underline{x}]$. Therefore, $s_{i}+Q=0+Q$, and $s_{i} \in R[\underline{x}] \cap Q=(0)$ for each $i$. Thus, $g=0$, and $R[u+y][\underline{x}] \cap Q=(0)$, and this implies that $S[\underline{x}] \cap Q=(0)$ for all $Q \in B_{1}$.

In order to use Proposition 2.1, we also must have that $I T \cap A=I$ for all finitely generated ideals $I$ of $A$. The following lemma, which follows the structure of [2, Lemma 2.6], will enable us to construct $A$ with this property.

Lemma 2.7. Let $(T, M)$ be a complete local ring of dimension at least one. Let $C$ be a countable set of nonmaximal prime ideals of $T$ such that if $U \in \operatorname{Ass} T$, then $U \subseteq P$ for some $P \in C$. Let $B_{1}$ be a set of prime ideals of $T[[\underline{x}]]$ with $\left|B_{1}\right|<|T|$ such that for all $Q \in B_{1}$, $Q \cap T \subseteq P$ for some $P \in C$. Let $R$ be an SCBA-subring of T. Suppose that $I$ is a finitely generated ideal of $R$ and $d \in I T \cap R$. Then there exists an SCBA-subring $S$ of $T$ such that $R \subseteq S \subseteq T, \Gamma(R)=\Gamma(S)$, and $d \in I S$.

Proof. We will induct on the number of generators of $I$. Suppose $I=a R$. Now if $a=0$, then $d=0$, and thus $S=R$ is the desired SCBA-subring of $T$. So consider the case where $a \neq 0$. In this case, 
$d=a u$ for some $u \in T$. We claim that $S=R[u]_{(R[u] \cap M)}$ is the desired SCBA-subring. To see this, first note that $\Gamma(R)=\Gamma(S)$, so $|S|<|T|$. Now let $f \in R[u] \cap P$ where $P \in C$. Then $f=$ $r_{n} u^{n}+\cdots+r_{1} u+r_{0} \in P$, with $r_{i} \in R$. Multiplying through by $a^{n}$, we get $a^{n} f=r_{n}(a u)^{n}+\cdots+r_{1} a^{n-1}(a u)+r_{0} a^{n}$, and it follows that $a^{n} f=r_{n} d^{n}+\cdots+r_{1} a^{n-1} d+r_{0} a^{n} \in P \cap R=(0)$. But $a \in R$, $R \cap P=(0)$ for every $P \in C$, and all associated prime ideals of $T$ are contained in an element of $C$. It follows that $a$ is not a zero divisor in $T$, yielding $f=0$, and $R[u]_{(R[u] \cap M)} \cap P=(0)$ for all $P \in C$.

We also show that $S[\underline{x}] \cap Q=(0)$ for all $Q \in B_{1}$. If $Q \in$ $B_{1}$ and $g \in R[u][\underline{x}] \cap Q=R[\underline{x}][u] \cap Q$, then $g=r_{n} u^{n}+\cdots+$ $r_{1} u+r_{0} \in Q$, where $r_{i} \in R[\underline{x}]$. Multiplying through by $a^{n}$, we get $a^{n} g=r_{n}(a u)^{n}+\cdots+r_{1} a^{n-1}(a u)+r_{0} a^{n}$, and it follows that $a^{n} g=r_{n} d^{n}+\cdots+r_{1} a^{n-1} d+r_{0} a^{n} \in Q \cap R[\underline{x}]=(0)$. Now, as $a$ is not a zerodivisor in $T$, it is also not a zerodivisor in $T[[\underline{x}]]$. It must be the case then that $g=0$, giving us that $S$ is the desired SCBA-subring of $T$.

Now let $I$ be an ideal of $R$ that is generated by $m>1$ elements, and suppose that the lemma holds true for all ideals of $R$ generated by $m-1$ elements. Let $I=\left(y_{1}, \ldots, y_{m}\right) R$, with $y_{1}, \ldots, y_{m}$ a minimal generating set for $I$. Then $d=y_{1} t_{1}+y_{2} t_{2}+\cdots+y_{m} t_{m}$ for some $t_{1}, t_{2}, \ldots, t_{m} \in T$. By adding 0 , we have the equality $d=y_{1} t_{1}+y_{1} y_{2} t-y_{1} y_{2} t+y_{2} t_{2}+$ $\cdots+y_{m} t_{m}=y_{1}\left(t_{1}+y_{2} t\right)+y_{2}\left(t_{2}-y_{1} t\right)+y_{3} t_{3}+\cdots+y_{m} t_{m}$ for any $t \in T$. Let $x_{1}=t_{1}+y_{2} t$ and $x_{2}=t_{2}-y_{1} t$, where we will choose the element $t$ later.

Now, let $P \in C$. If $\left(t_{1}+y_{2} t\right)+P=\left(t_{1}+y_{2} t^{\prime}\right)+P$, then it must be the case that $y_{2}\left(t-t^{\prime}\right) \in P$. But $y_{2} \in R, R \cap P=(0)$ and $y_{2} \neq 0$, so we have $t-t^{\prime} \in P$. Thus, $t+P=t^{\prime}+P$. The contrapositive of this result indicates that if $t+P \neq t^{\prime}+P$, then $\left(t_{1}+y_{2} t\right)+P \neq\left(t_{1}+y_{2} t^{\prime}\right)+P$. Similarly, for $Q \in B_{1}$, if $\left(t_{1}+y_{2} t\right)+Q=\left(t_{1}+y_{2} t^{\prime}\right)+Q$, then it must be the case that $y_{2}\left(t-t^{\prime}\right) \in Q$. But $y_{2} \in R[\underline{x}], R[\underline{x}] \cap Q=(0)$, and $y_{2} \neq 0$, so we have $t-t^{\prime} \in Q$, resulting in $t+Q=t^{\prime}+Q$. Again, the contrapositive of this result gives that if $t+Q \neq t^{\prime}+Q$, then $\left(t_{1}+y_{2} t\right)+Q \neq\left(t_{1}+y_{2} t^{\prime}\right)+Q$.

For each $P \in C$, let $D_{(P)}$ be a full set of coset representatives of the cosets $t+P$ that make $x_{1}+P$ algebraic over $R$. Similarly, for each $Q \in B_{1}$, define $D_{(Q)}$ to be a full set of coset representatives of 
the elements $t+(Q \cap T)$ in $T /(Q \cap T)$ that make $x_{1}+Q$ in $T[[\underline{x}]] / Q$ algebraic over $R[\underline{x}]$.

If we define $D=\left(\bigcup_{P \in C} D_{(P)}\right) \cup\left(\bigcup_{Q \in B_{1}} D_{(Q)}\right)$, then, by a similar argument to that in Lemma 2.6, we get that $|D|<|T|$. We can use Lemma 2.4 with $I=T$ to find an element $t \in T$ such that $x_{1}+P$ is transcendental over $R$ for every $P \in C$ and $x_{1}+Q$ is transcendental over $R[\underline{x}]$ for every $Q \in B_{1}$. It can easily be shown (as in the proof of Lemma 2.5) that $R^{\prime}=R\left[x_{1}\right]_{\left(R\left[x_{1}\right] \cap M\right)}$ is an SCBAsubring of $T$, with $\Gamma(R)=\Gamma\left(R^{\prime}\right)$. Now let $J=\left(y_{2}, \ldots, y_{m}\right) R^{\prime}$ and $d^{*}=d-y_{1} x_{1}$. Then $d^{*} \in J T \cap R^{\prime}$, so we can use our induction assumption to draw the conclusion that there exists an SCBA-subring $S$ of $T$ such that $R^{\prime} \subseteq S \subseteq T, \Gamma\left(R^{\prime}\right)=\Gamma(S)$, and $d^{*} \in J S$. Thus $d^{*}=y_{2} s_{2}+\cdots+y_{m} s_{m}$ for some $s_{2}, \ldots, s_{m} \in S$. It follows that $d=y_{1} x_{1}+y_{2} s_{2}+\cdots+y_{m} s_{m} \in I S$, and thus $S$ is the desired subring.

The following definition is used to tell whether an element of a wellordered set is a successor or a limit, which will be useful in constructing chains of SCBA-subrings.

Definition. Let $\Omega$ be a well-ordered set and $\alpha \in \Omega$. We define $\gamma(\alpha)=\sup \{\beta \in \Omega \mid \beta<\alpha\}$.

Lemma 2.8, based on [2, Lemma 2.7], allows us to construct SCBAsubrings with many of the properties we desire $A$ to possess.

Lemma 2.8. Let $(T, M)$ be a complete local ring of dimension at least one with prime subring $\Pi$. Let $C$ be a countable set of nonmaximal prime ideals of $T$ such that if $U \in \operatorname{Ass} T$, then $U \subseteq P$ for some $P \in C$. Let $J$ be an ideal of $T$ with $J \nsubseteq P$ for all $P \in C$. Let $B_{1}$ be a set of prime ideals of $T[[\underline{x}]]$ with $\left|B_{1}\right|<|T|$ such that if $Q \in B_{1}$ then $Q \cap T \subseteq P$ for some $P \in C$ and $Q \cap \Pi[\underline{x}]=(0)$. Let $u+J \in T / J$. Suppose $R$ is an SCBA-subring. Then there exists an infinite SCBAsubring $S$ of $T$ such that the following conditions hold:

(i) $R \subseteq S \subseteq T$.

(ii) $\Gamma(R)=\Gamma(S)$.

(iii) If $u \in J$, then $S \cap J \neq(0)$.

(iv) $u+J$ is in the image of the map $S \rightarrow T / J$.

(v) For every finitely generated ideal I of $S$, we have $I T \cap S=I$. 
This result follows directly from the proof of [2, Lemma 2.7], using Lemma 2.6 above in place of [2, Lemma 2.5] in the proof, with the exception of the second conclusion, which we must prove. We recreate the proof from [2] below, largely verbatim, making the necessary modifications.

Proof. First apply Lemma 2.6 to find an infinite SCBA-subring $R^{\prime}$ of $T$ such that $R \subseteq R^{\prime} \subseteq T, \Gamma(R)=\Gamma\left(R^{\prime}\right), u+J$ is in the image of the map $R^{\prime} \rightarrow T / J$, and if $u \in J$, then $R^{\prime} \cap J \neq(0)$. We will construct the desired $S$ such that $R^{\prime} \subseteq S \subseteq T$ and $\Gamma\left(R^{\prime}\right)=\Gamma(S)$ which will ensure that the first four conditions of the lemma hold true. Let

$$
\Omega=\left\{(I, d) \mid I \text { is a finitely generated ideal of } R^{\prime} \text { and } d \in I T \cap R^{\prime}\right\} .
$$

Letting $I=R^{\prime}$, we can see that $|\Omega| \geq\left|R^{\prime}\right|$. Conversely, since $R^{\prime}$ is infinite, the number of finitely generated ideals of $R^{\prime}$ is $\left|R^{\prime}\right|$, and therefore $\left|R^{\prime}\right| \geq|\Omega|$, giving us the equality $\left|R^{\prime}\right|=|\Omega|$. Moreover, as $R^{\prime}$ is an SCBA-subring, we have $|\Omega|=\left|R^{\prime}\right|<|T|$. Well order $\Omega$ so that it does not have a maximal element, and let 0 denote its first element. We will now inductively define a family of SCBA-subrings of $T$, one for each element of $\Omega$. Let $R_{0}=R^{\prime}$, and let $\alpha \in \Omega$. Assume that $R_{\beta}$ has been defined for all $\beta<\alpha$. If $\gamma(\alpha)<\alpha$ and $\gamma(\alpha)=(I, d)$, then define $R_{\alpha}$ to be the SCBA-subring obtained from applying Lemma 2.7 to $R_{\gamma(\alpha)}$ and our $C, B_{1}, I$, and $d$. In this manner, $R_{\alpha}$ will have the properties that $R_{\gamma(\alpha)} \subseteq R_{\alpha} \subseteq T, \Gamma\left(R_{\gamma(\alpha)}\right)=\Gamma\left(R_{\alpha}\right)$, and $d \in I R_{\alpha}$. If $\gamma(\alpha)=\alpha$, define $R_{\alpha}=\bigcup_{\beta<\alpha} R_{\beta}$. In this case, note that, for all $\beta<\alpha$, $\Gamma\left(R_{\beta}\right)=\Gamma\left(R^{\prime}\right)=\Gamma\left(R_{0}\right)$. Also note $|\{\beta \mid \beta<\alpha\}| \leq|\Omega|$.

Note that, in both cases, $R_{\alpha}$ is an SCBA-subring of $T$, with the property that $\Gamma\left(R_{\alpha}\right)=\Gamma\left(R_{0}\right)$. Now let $R_{1}=\bigcup_{\alpha \in \Omega} R_{\alpha}$. We know that $|\Omega|=\left|R^{\prime}\right|$ and $\Gamma\left(R_{\alpha}\right)=\Gamma\left(R^{\prime}\right)$ for every $\alpha \in \Omega$, so by Lemma 2.3, we know that $\Gamma\left(R_{1}\right) \leq \Gamma\left(R^{\prime}\right)$; but $R^{\prime} \subset R_{1}$ so $\Gamma\left(R_{1}\right)=\Gamma\left(R^{\prime}\right)$. Moreover, as $R_{\alpha} \cap P=(0)$ for every $P \in C$ and every $\alpha \in \Omega$, we have $R_{1} \cap P=(0)$ for every $P \in C$. Similarly, $R_{\alpha}[\underline{x}] \cap Q=(0)$ for every $Q \in B_{1}$ and for every $\alpha \in \Omega$. It follows that $R_{1}$ is an SCBA-subring. Furthermore, notice that if $I$ is a finitely generated ideal of $R_{0}$ and $d \in I T \cap R_{0}$, then $(I, d)=\gamma(\alpha)$ for some $\alpha \in \Omega$ with $\gamma(\alpha)<\alpha$. It follows from the

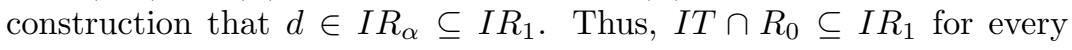
finitely generated ideal $I$ of $R_{0}$. 
Following this same pattern, build an SCBA-subring $R_{2}$ of $T$ such that $R_{1} \subseteq R_{2} \subseteq T, \Gamma\left(R_{1}\right)=\Gamma\left(R_{2}\right)$, and $I T \cap R_{1} \subseteq I R_{2}$ for every finitely generated ideal $I$ of $R_{1}$. Continue to form a chain $R_{0} \subseteq R_{1} \subseteq R_{2} \subseteq \cdots$ of SCBA-subrings of $T$ such that $I T \cap R_{n} \subseteq I R_{n+1}$ for every finitely generated ideal $I$ of $R_{n}$.

We now claim that $S=\bigcup_{i=1}^{\infty} R_{i}$ is the desired SCBA-subring. To see this, first note that $S$ is indeed an SCBA-subring, that $R \subseteq S \subseteq T$, and that $\Gamma\left(R^{\prime}\right)=\Gamma(S)$. Now set $I=\left(y_{1}, \ldots, y_{k}\right) S$, and let $d \in I T \cap S$. Then there exists an $N \in \mathbb{N}$ such that $d, y_{1}, \ldots, y_{k} \in R_{N}$. Thus, $d \in\left(y_{1}, \ldots, y_{k}\right) T \cap R_{N} \subseteq\left(y_{1}, \ldots, y_{k}\right) R_{N+1} \subseteq I S$. From this it follows that $I T \cap S=I$, so the fifth condition of the lemma holds.

Using Proposition 2.1, Lemma 2.9 provides a construction of a domain whose completion has the properties we desire. Properties 2 and 4 , which we have not mentioned yet, will be key in proving $A$ is excellent.

Lemma 2.9. Let $(T, M)$ be a complete local ring of dimension at least one with prime subring $\Pi$. Let $G$ be a set of nonmaximal prime ideals of $T$ such that $G$ contains the associated prime ideals of $T$ and the set of maximal elements of $G$, call it $C$, is countable. Let $B_{1}$ be a set of prime ideals of $T[[\underline{x}]]$ with $\left|B_{1}\right|<|T|$ such that if $Q \in B_{1}$, then $Q \cap T \subseteq P$ for some $P \in C$ and $Q \cap \Pi[\underline{x}]=(0)$. Let $B_{2}$ be a set of prime ideals of $T[[\underline{x}]]$, with $\left|B_{2}\right|<|T|$. Suppose also that there exists a set $W \subseteq T$ such that each ideal in $B_{2}$ contains a nonzero polynomial with coefficients in $W$. Also suppose that $\Pi[W] \cap P=(0)$ for all $P \in G$, and $\Pi[W][\underline{x}] \cap Q=(0)$ for all $Q \in B_{1}$. Moreover, suppose that if $p \in \operatorname{Spec} T$ with $p \subseteq P$ for some $P \in G$ then $p \in G$. Also suppose that for each prime ideal $P \in G, P$ contains no nonzero integers of $T$. Then there exists a local domain $A$ such that

(i) $\widehat{A}=T$.

(ii) If $p$ is a nonzero prime ideal of $A$, then $T \otimes_{A} k(p) \cong k(p)$ where $k(p)=A_{p} / p A_{p}$.

(iii) The generic formal fiber of $A$ is exactly the elements of $G$, having maximal elements the elements of $C$.

(iv) If $I$ is a nonzero ideal of $A$, then $A / I$ is complete.

(v) The elements of $B_{1}$ are contained in the generic formal fiber of $A[\underline{x}]_{(M \cap A, \underline{x})}$. And 
(vi) The elements of $B_{2}$ are not contained in the generic formal fiber of $A[\underline{x}](M \cap A, \underline{x})$.

Much of this proof comes from the construction of [2, Lemma 2.8], with several key distinctions. As such, that proof is constructed below, largely identically to its form in [2], but adapted as necessary.

Proof. First observe that, since $\left|B_{2}\right|<|T|$ and a polynomial has finitely many coefficients, we can reduce the size of $W$ if necessary to achieve $|W|<|T|$ while still maintaining the condition that, if $Q \in B_{2}$, then $Q$ contains a nonzero polynomial with coefficients in $W$. Also, reducing the size of $W$ will not make any other hypotheses of this lemma false unless they were false already. Denote this reduction of $W$ by $W^{\prime}$, and note that $\left|W^{\prime}\right|<|T|$.

Next define

$$
\Omega=\{u+J \in T / J \mid J \text { an ideal of } T \text { with } J \nsubseteq P \text { for all } P \in G\} .
$$

We claim that $|\Omega| \leq|T|$. We define $\Lambda=\{J \mid J$ is an ideal of $T$ with $J \nsubseteq P$ for all $P \in G\}$. Since $T$ is infinite and Noetherian, $|\Lambda| \leq|T|$. For any ideal $J$ of $T,|T / J| \leq|T|$. Observe $\Omega=\bigcup_{J \in \Lambda} T / J$, and so by Lemma 2.3, we see $|\Omega| \leq|T|$.

Well order $\Omega$ so that each element has fewer than $|\Omega|$ predecessors. Let 0 denote the first element of $\Omega$. Let $R_{0}^{\prime}=\Pi\left[W^{\prime}\right]$, and let $R_{0}$ be equal to $R_{0}^{\prime}$ localized at $R_{0}^{\prime} \cap M$. Thus, $R_{0}$ is a SCBA-subring.

Now, recursively define a family of SCBA-subrings as follows, starting with $R_{0}$. Let $\lambda \in \Omega$ and assume that $R_{\beta}$ has already been defined for all $\beta<\lambda$. Then $\gamma(\lambda)=u+J$ for some ideal $J$ of $T$ with $J \nsubseteq P$ for all $P \in G$ and thus for all $P \in C$. If $\gamma(\lambda)<\lambda$, use Lemma 2.8 to obtain an SCBA-subring $R_{\lambda}$ such that $R_{\gamma(\lambda)} \subseteq R_{\lambda} \subseteq T$, $u+J \in \operatorname{Image}\left(R_{\lambda} \rightarrow T / J\right)$, and for every finitely generated ideal $I$ of $R_{\lambda}$ the property $I T \cap R_{\lambda}=I$ holds. If $\gamma(\lambda)=\lambda$, define $R_{\lambda}=\bigcup_{\beta<\lambda} R_{\beta}$. Then we have $R_{\lambda}$ is a SCBA-subring for all $\lambda \in \Omega$. We claim that $A=\bigcup_{\lambda \in \Omega} R_{\lambda}$ is the desired domain.

From [2, Lemma 2.8], we know that conclusions (i)-(iv) are satisfied. Thus, we just need to show that the generic formal fiber of $A[\underline{x}]_{(M \cap A, \underline{x})}$ contains all elements of $B_{1}$ and no elements of $B_{2}$. Since each $R_{\lambda}$ 
is an SCBA-subring, $R_{\lambda}[\underline{x}] \cap Q=(0)$ for all $Q \in B_{2}$, and therefore $A[\underline{x}]_{(M \cap A, \underline{x})} \cap Q=(0)$ for each $Q \in B_{1}$.

Note that if $Q \in B_{2}$, then $Q$ contains a nonzero polynomial with coefficients in $W^{\prime}$, and therefore in $A$. This polynomial is in $Q \cap$ $A[\underline{x}]_{(M \cap A, \underline{x})}$, so the intersection is non-empty and no prime ideals in $B_{2}$ are in the generic formal fiber of $A[\underline{x}]_{(M \cap A, \underline{x})}$, as desired.

3. Main theorem. Using the last several lemmas, we are now able to construct a local domain $A$ that possesses the properties we desire.

Theorem 3.1. Let $(T, M)$ be a complete local ring with prime subring $\Pi$. Let $G$ be a set of prime ideals of $T$ such that the set of maximal elements of $G$, which we call $C$, is countable. Let $B_{1}$ and $B_{2}$ be sets of prime ideals of $T[[\underline{x}]]$, each with cardinality strictly smaller than $|T|$. Then there exists a local domain $A$ such that $\widehat{A}=T$, the generic formal fiber of $A$ is exactly the elements of $G$, the generic formal fiber of $A[\underline{x}]_{(M \cap A, \underline{x})}$ contains every ideal in $B_{1}$ and no ideal in $B_{2}$ if and only if the following conditions hold:

(i) Either $T$ is a field and $G=\{0\}$, or

(a) $M \notin G$, and $G$ contains all the associated prime ideals of $T$.

(b) If $Q \in G$ and $P \in \operatorname{Spec} T$ with $P \subseteq Q$, then $P \in G$.

(c) If $Q \in G$, then $Q \cap \Pi=(0)$.

(ii) If $Q \in B_{1}$, then $Q \cap T \in G$.

(iii) If $Q \in B_{1}$, then $Q \cap \Pi[\underline{x}]=(0)$.

(iv) There exists a subset $W \subset T$ such that

(a) If $Q \in B_{2}$ then there is a nonzero polynomial in $Q$ with coefficients in $W$.

(b) If $P \in C$ then $\Pi[W] \cap P=(0)$.

(c) If $Q \in B_{1}$ then $\Pi[W][\underline{x}] \cap Q=(0)$.

Proof. We will first prove that the four above conditions are necessary. Suppose there exists a local domain $A$ such that $\widehat{A}=T$, the generic formal fiber of $A$ is exactly $G$, and the generic formal fiber of $A[\underline{x}]_{(M \cap A, \underline{x})}$ contains all elements of $B_{1}$ and no elements of $B_{2}$. The necessity of the first condition follows from the fact that this theorem is a generalization of [ 1 , Theorem 2.13], and the first condition is necessary there. 
We will now prove that, for all $Q \in B_{1}, Q \cap T \subseteq P$ for some $P \in C$. First note that $A \subseteq A[\underline{x}]_{(M \cap A, \underline{x})} \subseteq T[[\underline{x}]]$. We know that $Q$ is in the generic formal fiber of $A[\underline{x}]_{(M \cap A, \underline{x})}$, and so $Q \cap A[\underline{x}]_{(M \cap A, \underline{x})}=(0)$. It follows that $Q \cap A=Q \cap A[\underline{x}]_{(M \cap A, \underline{x})} \cap A=(0)$. But $Q \cap A=(Q \cap T) \cap A$ so $Q \cap T$ is in the generic formal fiber of $A$, i.e. $Q \cap T \in G$.

We also know that, since $T$ is the completion of $A$, the unity element of $A$ must be in $T$, so $A$ and $T$ have the same prime subring. This means that $\Pi[\underline{x}]$ is necessarily in $A[\underline{x}]_{(M \cap A, \underline{x})}$. Therefore, since $Q \cap A[\underline{x}]_{(M \cap A, \underline{x})}=(0)$ for all $Q \in B_{1}$, we know that $Q \cap \Pi[\underline{x}]=(0)$.

For the fourth condition, $W=A$ is a set of the required form. We know $Q \cap A[\underline{x}]_{(M \cap A, \underline{x})} \neq(0)$ for all $Q \in B_{2}$, so there is a nonzero element of $Q$ that is also in $A[\underline{x}]_{(M \cap A, \underline{x})}$. An arbitrary element of $A[\underline{x}]_{(M \cap A, \underline{x})}$ looks like $z=f(\underline{x}) / g(\underline{x})$ for $f(\underline{x}), g(\underline{x}) \in A[\underline{x}] \subset T[[\underline{x}]]$. Thus, if $z \in \bar{Q}$, then $f(\underline{x}) \in Q$ as well, by the strong closure property of ideals. As $f(\underline{x}) \in A[\underline{x}]_{(M \cap A, \underline{x})}$, all the coefficients of $f(\underline{x})$ are in $A$, as desired. Furthermore, since $\Pi$ is a subring of $A$, we have $\Pi[W]=\Pi[A]=A$. The fact that the generic formal fiber of $A$ is exactly the elements of $C$ verifies condition (iv) (b); the fact that the generic formal fiber of $A[\underline{x}]_{(M \cap A, \underline{x})}$ contains every element of $B_{1}$ verifies (iv) (c). Thus, as desired, all four of the given conditions are necessary.

We will now prove the sufficient direction. Suppose the above four conditions are satisfied. Either $T$ is a field with $G=\{(0)\}$, in which case $A=T$ is the desired domain, or we can apply Lemma 2.9 (since (i) (a) implies the dimension of $T$ is at least one) to construct the desired domain $A$.

Consider the following very simple application of the preceding theorem.

Example 3.2. Let $T=\mathbb{Q}[[w, y, z]]$, and thus $T[[x]]=\mathbb{Q}[[x, w, y, z]]$. Let

$$
\begin{aligned}
& C=\{\langle y\rangle\}, \\
& G=\{\langle 0\rangle,\langle y\rangle\}, \\
& B_{1}=\{\langle y\rangle\}, \\
& B_{2}=\{\langle z x+w\rangle\}, \\
& W=\{w, z\} .
\end{aligned}
$$


We show that $T, G, B_{1}$, and $B_{2}$ satisfy the hypotheses of Theorem 3.1. By construction, the entire first hypothesis is satisfied. The second hypothesis is obviously true by our choice of $B_{1}$, since $Q \cap T=\langle y\rangle$ for all $Q \in B_{1}$. The third condition is easily satisfied as well, as $\Pi[x]$ contains only polynomials with integer coefficients in $x$. Clearly, $y$ does not divide any such nonzero polynomial, and $y$ divides every element of $\langle y\rangle$, so $\Pi[x] \cap\langle y\rangle=(0)$.

Finally note that (iv) (a) is satisfied by construction, and (iv) (b) and (iv) (c) are both satisfied in a similar way as the previous prime subring conditions. Thus, the given sets satisfy the four hypotheses of Theorem 3.1, and so we can construct a local domain $A$ whose completion is $T$, such that $A$ has a local generic formal fiber with maximal element $\langle y\rangle$. Furthermore, we can simultaneously ensure that the generic formal fiber of $A[x]_{(M \cap A, x)}$ contains $\langle y\rangle$ but does not contain $\langle z x+w\rangle$.

The following example is a better illustration of the level of control we have over the generic formal fibers of $A$ and $A[\underline{x}]_{(M \cap A, \underline{x})}$.

Example 3.3. Let $T=\mathbb{Q}\left[\left[y_{1}, y_{2}, z_{1}, z_{2}, z_{3}, z_{4}\right]\right]$, and so $T[[x]]=$ $\mathbb{Q}\left[\left[x, y_{1}, y_{2}, z_{1}, z_{2}, z_{3}, z_{4}\right]\right]$. Let

$$
\begin{aligned}
& C=\left\{\left\langle y_{1}+y_{2}^{k}\right\rangle \mid k \in \mathbb{Z}^{+}\right\}, \\
& G=C \cup\{\langle 0\rangle\}, \\
& E=\left\{\left\langle z_{1} x+z_{2}^{k}\right\rangle \mid k \in \mathbb{Z}^{+}\right\}, \\
& F=\left\{\left\langle z_{3} x+z_{4}^{k}\right\rangle \mid k \in \mathbb{Z}^{+}\right\}, \\
& W_{E}=\left\{z_{1}, z_{2}, z_{2}^{2}, z_{2}^{3}, \ldots\right\}, \\
& W_{F}=\left\{z_{3}, z_{4}, z_{4}^{2}, z_{4}^{3}, \ldots\right\} .
\end{aligned}
$$

Note first that $T$ is a complete local ring with maximal ideal $M=$ $\left\langle y_{1}, y_{2}, z_{1}, z_{2}, z_{3}, z_{4}\right\rangle$. The conditions are not difficult to verify, so we can use Theorem 3.1.

Applying Theorem 3.1 with $B_{1}=E, B_{2}=F, W=W_{F}$ results in a local domain $A$ such that $\widehat{A}=T$, the generic formal fiber of $A$ is exactly the elements of $G$, every element of $E$ is in the generic formal fiber of $A[x]_{(M \cap A, x)}$, and no element of $F$ is in the generic formal fiber of $A[x]_{(M \cap A, x)}$. 
Interestingly, we can also apply Theorem 3.1 with $B_{1}=F, B_{2}=E$, $W=W_{E}$, which results in a local domain $A$ such that $\widehat{A}=T$, the generic formal fiber of $A$ is exactly the elements of $G$, every element of $F$ is in the generic formal fiber of $A[x]_{(M \cap A, x)}$, and no element of $E$ is in the generic formal fiber of $A[x]_{(M \cap A, x)}$.

In the following corollary, all the conditions are the same except we substitute the condition $\left|B_{2}\right|<|T|$ with the condition $|W|<|T|$. While the new theorem gives us more control over $\left|B_{2}\right|$, we do not know that the condition $|W|<|T|$ is necessary.

Corollary 3.4. Let $(T, M)$ be a complete local ring with prime subring $\Pi$. Let $G$ be a set of nonmaximal prime ideals of $T$ such that the set of maximal elements of $G$, which we call $C$, is countable. Let $B_{1}$ and $B_{2}$ be sets of prime ideals of $T[[\underline{x}]]$ with $\left|B_{1}\right|<|T|$. Then there exists a local domain $A$ such that $\widehat{A}=T$, the generic formal fiber of $A$ is exactly the elements of $G$, the generic formal fiber of $A[\underline{x}]_{(M \cap A, \underline{x})}$ contains every ideal in $B_{1}$ and no ideal in $B_{2}$ if the following conditions are satisfied:

(i) Either $T$ is a field and $G=\{0\}$ or

(a) $M \notin G$, and $G$ contains all the associated prime ideals of $T$.

(b) If $Q \in G$ and $P \in \operatorname{Spec} T$ with $P \subseteq Q$, then $P \in G$.

(c) If $Q \in G$, then $Q \cap \Pi=(0)$.

(ii) If $Q \in B_{1}$ then $Q \cap T \in G$.

(iii) If $Q \in B_{1}$ then $Q \cap \Pi[\underline{x}]=(0)$.

(iv) There exists a subset $W \subset T$ such that

(a) $|W|<|T|$.

(b) If $Q \in B_{2}$ then there is a nonzero polynomial in $Q$ with coefficients in $W$.

(c) If $P \in C$ then $\Pi[W] \cap P=(0)$.

(d) If $Q \in B_{1}$ then $\Pi[W][\underline{x}] \cap Q=(0)$.

Proof. Since $|W|<|T|$, the proof of the sufficient direction of Theorem 3.1 also proves this corollary.

To illustrate when Corollary 3.4 is more powerful than Theorem 3.1, we give an example where $\left|B_{2}\right|=|T|$ but $|W|$ is finite. 
Example 3.5. Let $T=\mathbb{Q}\left[\left[y, z_{1}, \ldots, z_{6}\right]\right]$, and thus $T\left[\left[x_{1}, \ldots, x_{6}\right]\right]=$ $\mathbb{Q}\left[\left[x_{1}, \ldots, x_{6}, y, z_{1} \ldots, z_{6}\right]\right]$. Let

$$
\begin{aligned}
& C=\{\langle y\rangle\}, \\
& B_{1}=\{\langle y\rangle\}, \\
& I=\left\langle z_{1} x_{1}+z_{2} x_{2}\right\rangle, \\
& J=\left\langle z_{1} x_{1}+z_{2} x_{2}, z_{3} x_{3}+z_{4} x_{4}, z_{5} x_{5}+z_{6} x_{6}\right\rangle, \\
& B_{2}=\left\{Q \in \operatorname{Spec} T\left[\left[x_{1}, \ldots, x_{6}\right]\right] \mid I \subseteq Q \subseteq J\right\}, \\
& W=\left\{z_{1}, z_{2}\right\} .
\end{aligned}
$$

Note first that the prime subring of $T$ is simply $\mathbb{Z}$, and $I$ is a prime ideal of height 1 because it is generated by an irreducible polynomial. Also note that $J$ and $\left\langle z_{1} x_{1}+z_{2} x_{2}, z_{3} x_{3}+z_{4} x_{4}\right\rangle$ are both prime ideals of $T\left[\left[x_{1}, \ldots, x_{6}\right]\right]$. Since $\left\langle z_{1} x_{1}+z_{2} x_{2}, z_{3} x_{3}+z_{4} x_{4}\right\rangle$ is contained in $J$ and contains $I, J$ has height 3 . Call $R=T\left[\left[x_{1}, \ldots, x_{6}\right]\right] / I$ and $M=J / I$. Then $\left(T\left[\left[x_{1}, \ldots, x_{6}\right]\right] / I\right)_{(J / I)}=R_{M}$ is a local domain of dimension 2.

Suppose $\left|\operatorname{Spec} R_{M}\right|<\left|R_{M}\right|$. Note that $\left|R_{M}\right|=|R|=|R / M|=$ $\left|R_{M} / M R_{M}\right|$ because localizing does not change the cardinality of an infinite ring (first and third equalities), and $M$ is a non-maximal prime ideal of the complete local ring $R$ so Lemma 2.4 shows the second equality. Now $\left|\operatorname{Spec} R_{M}\right|<\left|R_{M} / M R_{M}\right|$ so we can use [4, Lemma 3] with $(T, M)=\left(R_{M}, M R_{M}\right), C=\operatorname{Spec} R_{M} \backslash\left\{M R_{M}\right\}, D=\{0\}$ and $I=M R_{M}$ to show that there is an $m \in M R_{M}$ such that $m$ is not contained in any prime ideal of $R_{M}$ except $M R_{M}$. But this is a contradiction, because $m$ is a non-unit in an integral domain, so it must be contained in some prime ideal of height one. Thus $\left|\operatorname{Spec} R_{M}\right| \nless\left|R_{M}\right|$.

The elements of $\operatorname{Spec}\left(T\left[\left[x_{1}, \ldots, x_{6}\right]\right] / I\right)_{(J / I)}=\operatorname{Spec} R_{M}$ correspond one-to-one with the prime ideals $Q$ of $T\left[\left[x_{1}, \ldots, x_{6}\right]\right]$ that satisfy $I \subseteq$ $Q \subseteq J$, which are exactly the elements of $B_{2}$. This shows $\left|B_{2}\right| \nless$ $\left|R_{M}\right|=\left|\left(T\left[\left[x_{1}, \ldots, x_{6}\right]\right] / I\right)_{(J / I)}\right|=\left|T\left[\left[x_{1}, \ldots, x_{6}\right]\right]\right|$. In particular, we cannot use Theorem 3.1 for this case, because $\left|B_{2}\right|=|T|$. But, clearly, $|W|<|T|$, so we can use Corollary 3.4 instead.

It is easy to verify the conditions on $P$ and $B_{1}$. This is an interesting example because $B_{2}$ also satisfies the conditions on $B_{1}$ : for any $Q \in B_{2}$, $Q \cap T \subseteq J \cap T \subseteq\left\langle x_{1}, \ldots, x_{6}\right\rangle \cap T=\langle 0\rangle \subset P$ and $Q \cap \mathbb{Z}\left[x_{1}, \ldots, x_{6}\right] \subseteq$ $J \cap \mathbb{Z}\left[x_{1}, \ldots, x_{6}\right] \subseteq\left\langle z_{1}, \ldots, z_{6}\right\rangle \cap \mathbb{Z}\left[x_{1}, \ldots, x_{6}\right]=\langle 0\rangle$. Thus, each ideal in $B_{2}$ can individually be put inside or outside the generic formal fiber of $A\left[x_{1}, \ldots, x_{6}\right]$. 
Now we show the rest of the conditions are satisfied. Condition (i) is true since for all $Q \in B_{2}, z_{1} x_{1}+z_{2} x_{2} \in Q$ and therefore $Q$ contains a non-zero polynomial with coefficients in $W$. Condition (ii) is satisfied as $\mathbb{Z}[W] \cap P=\mathbb{Z}\left[x_{1}, x_{2}\right] \cap\langle y\rangle=\langle 0\rangle$. And, condition (iii) holds since $\mathbb{Z}[W]\left[x_{1}, \ldots, x_{6}\right] \cap\langle y\rangle=\mathbb{Z}\left[x_{1}, \ldots, x_{6}\right] \cap\langle y\rangle=\langle 0\rangle$.

As shown above, all the conditions are satisfied, and the corollary tells us that there is a local domain $A$ such that $\widehat{A}=T$, the generic formal fiber of $A$ is local with maximal ideal $P$ and the generic formal fiber of $A\left[x_{1}, \ldots, x_{6}\right]$ contains $B_{1}$ but no element of $B_{2}$. Note that we can also construct $A$ to be an excellent ring, which we will show later in Section 4.

Consider now the following corollary, which only controls which elements of Spec $T[[\underline{x}]]$ belong to the generic formal fiber of $A[\underline{x}]_{(M \cap A, \underline{x})}$, but has much simpler conditions than Theorem 3.1.

Corollary 3.6. Let $(T, M)$ be a complete local ring with prime subring $\Pi$. Let $G$ be a set of nonmaximal prime ideals of $T$ such that the set of maximal elements of $G$, which we call $C$, is countable. Let $B_{1}$ be a set of prime ideals of $T[[\underline{x}]]$, with cardinality strictly smaller than $|T|$. Then there exists a local domain $A$ such that $\widehat{A}=T$, the generic formal fiber of $A$ is exactly the elements of $G$, and the generic formal fiber of $A[\underline{x}]_{(M \cap A, \underline{x})}$ contains every ideal in $B_{1}$ if and only if the following conditions are met:

(i) Either $T$ is a field and $G=\{0\}$ or

$M \notin G$, and $G$ contains all the associated prime ideals of $T$.

If $Q \in G$ and $P \in \operatorname{Spec} T$ with $P \subseteq Q$, then $P \in G$.

If $Q \in G$, then $Q \cap \Pi=(0)$.

(ii) If $Q \in B_{1}$ then $Q \cap T \in G$.

(iii) If $Q \in B_{1}$ then $Q \cap \Pi[\underline{x}]=(0)$.

Proof. Let $B_{2}=\emptyset$ and $W=\emptyset$, and apply Theorem 3.1.

4. Making $A$ excellent. We would like to understand when a complete local ring $(T, M)$ is the completion of an excellent local domain $A$ such that both the generic formal fiber of $A$ and $A[\underline{x}]_{(M \cap A, \underline{x})}$ can be controlled. To that end, we have the following result. 
Theorem 4.1. Let $(T, M)$ be a complete local ring of characteristic 0 with prime subring $\Pi$. Let $G$ be a set of prime ideals of $T$ such that the set of maximal elements of $G$, which we call $C$, is countable. Let $B_{1}$ and $B_{2}$ be sets of prime ideals of $T[[\underline{x}]]$, each with cardinality strictly smaller than $|T|$. Then there exists an excellent local domain $A$ such that $\widehat{A}=T$, the generic formal fiber of $A$ is exactly the elements of $G$, and the generic formal fiber of $A[\underline{x}]_{(M \cap A, \underline{x})}$ contains every ideal in $B_{1}$ and no ideal in $B_{2}$ if and only if the following conditions are met:

(i) Either $T$ is a field and $G=\{0\}$, or

(a) $M \notin G$, and $G$ contains all the associated prime ideals of $T$.

(b) If $Q \in G$ and $P \in \operatorname{Spec} T$ with $P \subseteq Q$, then $P \in G$.

(c) If $Q \in G$, then $Q \cap \Pi=(0)$.

(d) $T$ is equidimensional.

(e) $T_{P}$ is a regular local ring for all $P \in C$,

(ii) If $Q \in B_{1}$ then $Q \cap T \in G$.

(iii) If $Q \in B_{1}$ then $Q \cap \Pi[\underline{x}]=(0)$.

(iv) There exists a subset $W \subset T$ such that

(a) If $Q \in B_{2}$ then there is a nonzero polynomial in $Q$ with coefficients in $W$,

(b) If $P \in C$ then $\Pi[W] \cap P=(0)$,

(c) If $Q \in B_{1}$ then $\Pi[W][\underline{x}] \cap Q=(0)$.

Proof. This result follows directly from the proof of [2, Theorem 4.1], using Lemma 2.9 above in place of [2, Lemma 2.8] in the proof.

Note that, in the previous examples, all the conditions stated in the above theorem are satisfied. Therefore, the local domains $A$ constructed were indeed also excellent. It is worthwhile to give a concrete example of a case where such an excellent domain $A$ does not exist.

Example 4.2. Let $T=\mathbb{Q}\left[\left[y_{1}, y_{2}, z_{1}, z_{2}, z_{3}, z_{4}\right]\right] /\left\langle y_{1}^{2}, y_{2}^{2}\right\rangle$. Thus, we have $T[[x]]=\mathbb{Q}\left[\left[x, y_{1}, y_{2}, z_{1}, z_{2}, z_{3}, z_{4}\right]\right] /\left\langle y_{1}^{2}, y_{2}^{2}\right\rangle$.

Moreover, let

$$
\begin{aligned}
& C=\left\{\left\langle z_{1}+z_{2}^{k}, y_{1}, y_{2}\right\rangle \mid k \in \mathbb{Z}^{+}\right\} . \\
& B_{1}=\left\{\left\langle z_{1} x+z_{2}^{k}, y_{1}, y_{2}\right\rangle \mid k \in \mathbb{Z}^{+}\right\} . \\
& B_{2}=\left\{\left\langle z_{3} x+z_{4}^{k}, y_{1}, y_{2}\right\rangle \mid k \in \mathbb{Z}^{+}\right\} .
\end{aligned}
$$




$$
W=\left\{z_{3}, z_{4}^{k} \mid k \in \mathbb{Z}^{+}\right\} .
$$

Note that, unlike the previous examples, $T$ in this example is not a domain. However, Ass $T=\left\{\left\langle y_{1}, y_{2}\right\rangle\right\}$, which is contained in all elements of $C$. Therefore condition (i) (a) of Theorem 3.1 is satisfied. All other conditions of Theorem 3.1 can be checked similarly to Example 3.2, and therefore we can apply Theorem 3.1 to show a local domain $A$ exists, which need not be excellent. To see that $A$ can never be excellent, notice that we have nilpotent elements $y_{1}$ and $y_{2}$ in $T_{P}$ for all $P \in C$. But all regular rings are reduced, so $T_{P}$ cannot be regular local. Since $T$ fails to satisfy Theorem 4.1 (i) (e), an excellent local domain $A$ does not exist.

As in Corollary 3.6, if we give up the possibility of specifying prime ideals to be outside the generic formal fiber of $A[\underline{x}]_{(M \cap A, \underline{x})}$, then we can specify prime ideals to be in its generic formal fiber with much simpler conditions.

Corollary 4.3. Let $(T, M)$ be a complete local ring of characteristic 0 with prime subring $\Pi$. Let $G$ be a set of prime ideals of $T$ such that the set of maximal elements of $G$, which we call $C$, is countable. Let $B_{1}$ be a set of prime ideals of $T[[\underline{x}]]$ with cardinality strictly smaller than $|T|$. Then there exists an excellent local domain $A$ such that $\widehat{A}=T$, the generic formal fiber of $A$ is exactly the elements of $G$, and the generic formal fiber of $A[\underline{x}]_{(M \cap A, \underline{x})}$ contains $B_{1}$ if and only if the following conditions are met.

(i) Either $T$ is a field and $G=\{0\}$, or

(a) $M \notin G$, and $G$ contains all the associated prime ideals of $T$.

(b) If $Q \in G$ and $P \in \operatorname{Spec} T$ with $P \subseteq Q$, then $P \in G$.

(c) If $Q \in G$, then $Q \cap \Pi=(0)$.

(d) $T$ is equidimensional.

(e) $T_{P}$ is a regular local ring for all maximal elements.

(ii) If $Q \in B_{1}$, then $Q \cap T \subseteq P$ for some $P \in C$.

(iii) If $Q \in B_{1}$, then $Q \cap \Pi[\underline{x}]=(0)$.

Proof. Let $B_{2}=\emptyset$ and $W=\emptyset$, and apply Theorem 4.1. 


\section{REFERENCES}

1. Domenico Aiello, S. Loepp and Philip Vu, Formal fibers with countable many maximal elements, Rocky Mountain J. Math. 45 (2015), 371-388.

2. P. Charters and S. Loepp, Semilocal generic formal fibers, J. Alg. 278 (2004), $370-382$.

3. John Chatlos, Brian Simanek, Nathaniel G. Watson and Sherry X. Wu, Semilocal formal fibers of principal prime ideals, J. Comm. Alg. 4 (2012), 369385 .

4. Raymond C. Heitmann, Characterization of completions of unique factorization domains, Trans. Amer. Math. Soc. 337 (1993), 379-387.

5. , Completions of local rings with an isolated singularity, J. Alg. 163 (1994), 538-567.

6. S. Loepp and C. Rotthaus, Generic formal fibers of polynomial ring extensions, Rocky Mountain J. Math. 34 (2004), 253-262.

7. S. Loepp and Aaron Weinberg, Generic formal fibers of polynomial rings, J. Pure Appl. Alg. 163 (2001), 93-106.

8. Hideyuki Matsumura, Commutative ring theory, Cambridge University Press, Cambridge, 1986.

9. Christel Rotthaus, Excellent rings, Henselian rings, and the approximation property, Rocky Mountain J. Math. 27 (1997), 317-334.

Department of Mathematics, Brown University, Box 1917, 151 Thayer Street, Providence, RI, 02912

Email address: pj4@math.brown.edu

School of Mathematics, Georgia Institute of Technology, 686 Cherry Street, Atlanta, GA 30332

Email address: kirkpatrickae@gmail.com

18 Hoxsey St., Department of Mathematics and Statistics, Williams ColLEge, Williamstown, MA 01267

Email address: sloepp@williams.edu

2049 Martin Luther King Jr. Drive, Department of Mathematics, Applied Mathematics and Statistics, Case Western Reserve University, Cleveland, $\mathrm{OH} 44106$

Email address: mack-crane@case.edu

18 Hoxsey St., Department of Mathematics and Statistics, Williams ColLEge, Williamstown, MA 01267

Email address: samuel.tripp1@gmail.com 